\title{
Reply to: What Causes Late Perforation of the Jejuno-jejunal Anastomosis After Roux-en-Y Gastric Bypass Surgery?
}

\author{
David Goitein $^{1,2}$ (D)
}

Published online: 28 August 2015

(C) Springer Science+Business Media New York 2015

The authors report a case of late jejuno-jejunal (JJ) anastomotic perforation 11 months following Roux-en$\mathrm{Y}$ gastric bypass. As they correctly state, this is a rare phenomenon, occurring in less than $1 \%$ of cases. It is therefore sporadically reported. It is possible, though unlikely, that it is an under-reported event. Being such a rare occurrence, it is difficult, if not impossible, to draw meaningful conclusions as to its cause, and all attempts are purely speculative.

Common general surgical practice teaches us that small to small bowel anastomoses are among the safest of possible intestinal connections. This is due to the excellent small intestinal blood supply, the anatomic construct of the small bowel wall, and ample mobility, preventing tension. They are therefore not prone to perforate late in the postoperative course, and this is true in bariatric surgery as well.

As to the second question posed - in any of the cases reported-ulceration (ischemic or other) was not mentioned as the root of the perforation. Ischemia, as the cause of anastomotic breakdown, occurs within 5-
10 days and not a likely cause in the late phase. Mesenteric events, thromboembolic or low-flow related, may happen anywhere along the small bowel, but would not "target" the JJ site. Again, in all reported cases, mesenteric ischemia was not implied.

The change in gut flora following bariatric surgery has been recently implied as a possible contributory mechanism for the metabolic effect of these procedures. There is indeed a "shift" of the gut microbiota from an "obese" to a "lean" distribution. It is doubtful that this change could be the cause of late JJ perforation.

To conclude, late perforation of the $\mathrm{JJ}$ anastomosis is a very rare complication. No specific cause or risk factor has yet been found, but awareness of this potentially lethal complication is important to both bariatric and general surgeons.

Conflict of Interest David Goitein declares that he has no competing interests.

Ethical Approval For this type of paper formal consent is not required.

Informed Consent Does not apply.

David Goitein

david.goitein@sheba.health.gov.il

1 Bariatric \& Metabolic Surgery, Dept. of Surgery C, Chaim Sheba Medical Center, Tel Hashomer 52621, Israel

2 Sackler School of Medicine, Tel Aviv University, Tel Aviv, Israel 